Horizons philosophiques

\title{
Michel Serres et le vrai nom de Dieu
}

\section{Claude Lagadec}

Volume 8, numéro 1, automne 1997

Le Monde de Michel Serres

URI : https://id.erudit.org/iderudit/801059ar

DOI : https://doi.org/10.7202/801059ar

Aller au sommaire du numéro

Éditeur(s)

Collège Édouard-Montpetit

ISSN

1181-9227 (imprimé)

1920-2954 (numérique)

Découvrir la revue

Citer cet article

Lagadec, C. (1997). Michel Serres et le vrai nom de Dieu. Horizons

philosophiques, 8(1), 41-54. https://doi.org/10.7202/801059ar d'utilisation que vous pouvez consulter en ligne.

https://apropos.erudit.org/fr/usagers/politique-dutilisation/ 


\section{MICHEL SERRES ET LE VRAI NOM DE DIEU}

Quiconque s'intéresse à la philosophie connaît le fossé qui sépare de nos jours la philosophie qui s'enseigne dans nos écoles, du savoir qui se construit par ailleurs sous le nom de science. II n'y a rien de commun, en effet, entre Platon et Marie Curie, entre Gilles Deleuze et la biologie moléculaire ou entre Nietzsche et l'astrophysique. En pratique il y a là deux mondes, on l'a assez dit, deux cultures qui s'ignorent, généralement sans appel. Dans les cinq ouvrages qui composent la série Hermès Michel Serres cherche à combler ce fossé, à provoquer les retrouvailles de ces deux mondes dans ce qu'il appelle la Communication.

II rêve de l'époque heureuse où la science et l'humanisme faisaient bon ménage dans un ensemble accueillant aussi bien le mythe, les récits édifiants, les contes et légendes. Jusqu'à un certain point ce désir est partagé par un grand nombre de philosophes de notre époque conscients de l'effritement progressif mais apparemment irrésistible de la distinction radicale entre les faits et les valeurs. La science moderne s'était édifiée sur la prétention de ne traiter que des faits, à l'exclusion des valeurs abandonnées aux philosophes et théologiens. Chacun peut reconnaître de nos jours que les propositions portant sur les faits supposent elles-mêmes des valeurs. Mais personne n'est allé aussi loin que Michel Serres pour faire circuler et communiquer les divers antagonismes séparant la philosophie de la littérature et de la science, et en cela l'entreprise de Serres est la nôtre à tous.

La méthode de Michel Serres est une anti-méthode, plus proche de l'application que de l'explication, qui permettrait au philosophe et au scientifique de reprendre possession de l'entièreté de leur boîte à outils commune. Jusqu'au XVIIIe siècle la science c'était la philosophie au même titre que le calcul, la religion, l'art, le mythe. Voyez comme c'est grand dehors, dit-il, pensez grand, visez loin, «le savoir naît heureux ». Le maître 
dont Michel Serres s'inspire principalement est Gottfried Wilhelm Leibniz, philosophe mathématicien pour qui l'existence de Dieu est parfaitement démontrable rationnellement. À son époque le vrai nom de Dieu était Raison. De nos jours le vrai nom de Dieu est Communication. Surfez.

Qu'advient-il à cette recherche d'un terrain commun entre les sciences exactes et les sciences humaines? L'entreprise est représentée sous la métaphore du passage du Nord-Ouest. La réponse finale apparaît dans les premières pages du dernier tome de la série des Hermès. Le Passage du Nord-Ouest reconnaît que le chemin n'est pas aussi simple qu'on aurait pu penser : "L'important est ici de dire que le passage est rare et resserré [...] Le plus souvent, le passage est fermé, soit par terres, soit par glaces, soit aussi parce qu'on se perd. Et si le passage est ouvert, c'est le long d'un chemin difficile à prévoir». La possibilité d'un échec est calmement examinée. II se peut, dit l'auteur, que l'extraordinaire succès remporté par Newton transposant la loi de la chute des corps à la circulation des planètes, donc du local au global, soit un cas unique qui ne se représentera pas. "Cette chance ne se présente pas tous les matins. Ce que nous avons usage de nommer la raison, la rationalité, n'est, peut-être, qu'un cas rare. Le rationnel serait un îlot plongé dans le réel». On entrevoit, ici, la hardiesse de l'entreprise en même temps que la simplicité que l'auteur met à en reconnaître la difficulté. Nous savons, dit-il, que le travail de l'historien, par exemple, demeure toujours fondamentalement différent de celui du savant. L'historien travaille dans le positif, il accumule des données qu'il s'efforce de mettre en relation, il n'écarte a priori aucun phénomène et s'inquiète d'avoir oublié quelque chose. Son travail n'est jamais falsifiable, il œuvre dans l'universel auquel rien, en principe, n'est étranger. Le savant, au contraire, travaille dans le particulier, il finit toujours par vous dire que ce dont vous parlez ne l'intéresse pas. Je suis physicien, dira-t-il, et je n'ai rien à dire sur la sociologie électorale. Le savant travaille par exclusions successives, il élimine, soustrait, exclut. II travaille dans le négatif. 
Parfois, cependant, arrivant d'ailleurs, un inventeur particulier parvient à se frayer un passage. "L'inventeur est d'ailleurs, il fait de l'ailleurs". Donc, tout en demeurant difficultueux le passage n'est pas impossible et j'en témoigne, dit Serres : «Des passages existent, j'en sais, j'en ai rangés, en certaines œuvres, par de certains opérateurs. Le dernier fut le parasite1, où des sciences exactes aux humaines, culturel et naturel ensemble et d'une voix, la voie fut ouverte et cartographiée. Mais je ne puis généraliser". II est vrai que la tâche est immense, conclut-il, mais ce n'est vraiment pas la peine, jeune homme, de s'aventurer en philosophie «si l'on n'a pas l'espoir, le projet ou le rêve de tenter un jour la synthèse. Le moins qu'on puisse essayer en ces lieux est le tour d'un monde ou les douze travaux d'Héraclès. Au moins cela, quand on paie aussi cher en veille, étude et solitude».

Je voudrais pour ma part interroger le rôle de la mort dans la réponse donnée à la quête entreprise par la série des Hermès. Lidée de mort apparaît notamment dans Hermès III. La traduction, dont le troisième chapitre s'intitule "Trahison : la thanatocratie". II s'agit, dans un premier temps, de la menace nucléaire lourdement ressentie au temps de la guerre froide, à l'époque de la publication de La traduction. L'auteur voit dans le projet Manhattan l'aboutissement de plusieurs siècles d'asservissement de la science à des entreprises de pouvoir et de mort. II est question du "Ministère de la mort", de "l'instinct de mort", en même temps que la perte, par la science, de sa propre composante historique. Puis la question s'élargit, le ton monte encore, tempo mit grösser Vehemenz, la phrase s'enfle et approche l'imprécation pour associer la science et la rationalité elle-même à une pratique politique de domination et de mort. La science est vouée dès le départ du miracle grec, dit-il, à l'appropriation de la mort et de la destruction légitimes. "La raison est malade à mourir à l'instant de son émergence». L'aigreur passe au front du savant et l'ennui suinte des murs. L'analyse se poursuit sur le ton d'infinie tristesse de celui qui

1. Michel Serres, Le parasite, Paris : Bernard Grasset, 1980. 
voit «toute l'utilité du savoir, à peu près, canalisée vers la mort». Le thème de la mort, élargi à la critique, est repris sur un mode plus analytique dans Le passage du Nord-Ouest. Après avoir montré que le savant travaille dans le négatif, comme je l'ai dit plus haut, Serres ajoute :

Les querelles sont le frein de l'histoire et le moteur de la sottise. La libido dominandi exclut toute invention, toute intuition, toute découverte. Le nouveau est incomparable. Rien n'est plus vieux que la comparaison, rien n'est plus répétitif que la polémique, ni conservateur que le combat?2.

Les idéologies, les théories, les religions, les sciences nous ont toujours bercés d'espérance tant qu'elles remplissaient une fonction critique; elles ont toujours été atroces quand elles ont obtenu le pouvoir. Lucides et généreuses tout d'abord, implacables après. Cette loi n'a pas d'exception, nous sommes abondamment payés pour l'avoir apprise. Pourquoi voulez-vous que les sciences humaines soient, justement, cette exception?. [...] Tant que la science est l'objet d'un discours, le travail est une œuvre, un loisir, une distraction, presque un art; qu'elle devienne outil de pouvoir, la mort est là. Une place, alors, reste vide, et ceux qui l'occupent sont toujours absents. Là où se pose la question la plus banale et la plus rare, la plus pressante et la plus évitée : pourquoi? ${ }^{3}$

\section{La question la plus banale et la plus rare, la plus pressante et la plus évitée}

Voilà la question à laquelle je voudrais tenter de répondre maintenant: Pourquoi le pouvoir s'approprie-t-il le savoir? Puisqu'il est question de mort, il va forcément être question de vie. Je propose de reprendre à nouveaux frais, et risques, la question posée par Serres. Je remplacerai, dans la suite de ce texte, la métaphore du passage d'un océan à un autre par celle de la construction d'un pont au-dessus d'un vide qui est de notre fait. Le vide, ou la différence, semble être en nous et nous

2. Hermès IV : Le passage du Nord-Ouest, Paris : Édition de Minuit, 1980, p.102.

3. Ibid., p.125. 
y avons sans cesse recours dans nos raisonnements, depuis au moins les Grecs, chaque fois que l'intelligible est dit incomparable au sensible, chaque fois que nous affirmons être un composé d'un corps et d'une âme et qu'il existe d'une part des sujets et d'autre part des objets. Le pont dont nous avons besoin rendrait possible le passage entre les deux rives qui bordent ce vide, sans pour autant autoriser le ridicule de prétendre l'abolir. II y a un vide et nous avons besoin d'un pont. En clair, la construction proposée n'est pas matérialiste. J'ai toujours pensé le matérialiste pas très sérieux qui prétend dire, avec des mots, que les mots sont sans importance puisque seule la matière importe. Qu'il le dise alors avec la matière! Le philosophe demeure dualiste tant qu'il utilise des mots pour dire des choses. Le langage, à lui seul, creuse le vide qui me fend et le comble quand je dis. C'est sans doute pourquoi le savoir naît heureux. Ce qui nous sépare de nous-mêmes, je de me et tu de nous, est cela même qui peut nous réunir. La construction du pont ne sera donc qu'une réappropriation après beaucoup d'autres, autrefois on aurait dit désaliénation mais il n'y a pas place pour la désaliénation dans la philosophie à plusieurs voix. Plusieurs triomphe. Le va-et-vient sur le pont sera l'eurythmie du monde. On trouve quelques-uns des matériaux requis pour la construction du pont dans l'évolution de la vie :

1. L'évolution. Nous sommes des organismes produits par une évolution opportuniste et sans but. La vie est tout d'abord de l'ordre au sens de la thermodynamique, donc entropie négative et information. Serres reprend l'exemple d'Aristote, un tas de pierres n'est pas une maison mais à long terme la maison produit un tas de pierres. Une maison c'est de l'ordre, un produit de la vie. Cet ordre n'est que la première moitié de l'évolution, l'autre étant la sélection naturelle décrite par Darwin. Ce qui fait que l'évolution ne peut être dite ni déterministe ni indéterministe et l'on peut légitimement soutenir qu'elle est les deux à la fois. Elle est déterministe dans le sens où le génome est un ensemble clos qui se transmet par des mécanismes aux effets prévisibles, comme les lois de Mendel. Mais elle est aussi indéterministe, et ceci en deux sens distincts : a) d'abord la pro- 
duction, puis la transmission du matériel génétique, occasionnent des écarts aléatoires qui sont producteurs de mutants; $b$ ) par la suite chaque phénotype est soumis à une sélection dans un environnement essentiellement changeant. L'évolution est le produit de ces deux processus, d'abord déterministe puis indéterministe. Le résultat est que, contrairement à la mécanique de Newton qui nous permet de déterminer la position des planètes dans l'avenir comme dans le passé, en évolutionnisme on ne peut rien prédire, pour les mêmes raisons de complexité évoquées par Serres parlant de météorologie : dans les deux cas, évolutionnisme ou météorologie, le nombre de facteurs à intégrer est simplement trop grand pour autoriser la prédiction à long terme. C'est pourquoi, en évolutionnisme, l'expression "déterminisme biologique» sans quantificateur que l'on ne trouve jamais chez Serres - est un non-sens à l'égal de la quadrature du cercle. II n'y a pas déterminisme là où l'on ne peut pas prédire. Le primatologue Hans Kummer le fait comprendre par une élégante comparaison entre le couple génétique-environnement et la musique. II serait vain, dit-il, de demander à savoir si la musique procède davantage de l'exécutant que de l'instrument, tout ce que l'on peut dire c'est qu'avec un autre batteur ou avec un autre tambour, la musique serait différente 4 . C'est ce double caractère déterministeindéterministe de l'évolution ou, si l'on veut, le fait de n'être ni déterministe ni indéterministe et proche du tiers-inclus qu'affectionne Michel Serres, qui a fait l'extraordinaire fécondité de l'évolutionnisme de Darwin. Aristote nous avait montré que le même cause le même, l'homme engendre l'homme et le pommier produit des pommes. Darwin, lui, nous montre comment le même cause l'autre, comment la faune marine a engendré le serpent qui a engendré l'oiseau et le mammifère et le primate parlant. L'extraordinaire est que l'évolution a inventé le langage, la nature a engendré la culture. Quand le même cause l'autre, tout est possible, par bon vent.

4. Cité dans Linda Marie Fedigan, Primate Paradigms, Montréal : Eden Press, 1982, p. 31. 
2. Différents. Nous sommes génétiquement uniques. Sauf rares exceptions, c'est la règle pour les organismes engendrés sexuellement. Le fait d'être unique au monde rend chacun de nous totalement différent de tout autre être humain. Le brassage génétique qui précède et accompagne la fécondation est extrêmement élevé. F.J. Ayala 5 a calculé que le nombre de cellules sexuelles différentes possibles, chez l'homme, est le produit de 2 à la puissance 6700 par 10 à la puissance 2 017. Ce nombre est beaucoup plus élevé, dit-il, que le nombre d'atomes dans l'univers connu qui est d'environ 1080. Plusieurs humains ont eu l'occasion au moins une fois, au cours de leur existence, de se poser la question : "Qu'est-ce que j'ai bien pu faire au bon Dieu pour avoir - ou avoir été engendré par ce monstre?" Nous sommes tous uniques et différents.

3. La société d'inégalité. Nous sommes des primates sociaux et non pas solitaires. L'évolution produit des organismes des deux sortes. Parmi les 20000 espèces d'abeilles, par exemple, la plupart sont solitaires, quelques-unes seulement sont devenues sociales. Chez nos ancêtres c'est plutôt le contraire qui s'est passé, la plus grande partie des quelque 200 espèces de primates, dont la nôtre, sont sociales à un degré ou un autre. La vie sociale combine l'égoïsme de la lutte pour l'existence, en quoi nous sommes égaux et qui est la règle en biologie solitaire, avec la coopération. La vie sociale est un mixte qui entraîne la division du travail particulière à l'espèce, l'accès à la nourriture, la répartition des rôles selon les sexes, le mode de reproduction, et ainsi de suite. Or j'ai dit que nous sommes différents. II se trouve que la distribution de ces différences à l'intérieur de l'ensemble social prend une forme stochastique, ce qui est l'équivalent de l'établissement de normes de facto avantageant certains points particuliers le long de la courbe statistique, aux dépens de certains autres points. Nous sommes également égoïstes mais nos différents égoïsmes sont inégalement récompensés. La sélection naturelle opérant dans la vie sociale comme ailleurs, le nombre des candidats aux postes

5. Francisco J. Ayala, "The mechanisms of Evolution", Scientific American, vol. 239,1978 , p. 56-59. 
clés requis par la coopération a donc de fortes chances de toujours excéder celui des postes disponibles. Le tri évolutionniste qui s'ensuit institue la hiérarchie sociale propre à l'espèce.

La description suivante résume des recherches répétées sur de nombreuses espèces, elle est devenue canonique et a dorénavant valeur générale, avec les variantes propres à chaque espèce, évidemment. Carl Murchison ${ }^{6}$ a montré que la chaîne de dominance d'un groupe de coqs et leur consommation de nourriture sont tout d'abord faibles, les volailles s'agressant fréquemment. Avec le temps un ordre de dominance tend à s'instaurer. À partir de la $32^{\mathrm{e}}$ semaine de vie commune l'ordre devient total, linéaire. Désormais les agressions ouvertes se raréfient, les inférieurs sociaux défèrent devant les dominants qui ritualisent les signes de leur rang, la consommation de nourriture s'optimalise dans une paix sociale relativement stable.

Remarque. Nous savons qu'on ne peut jamais transposer inconsidérément, terme à terme, les données de la biologie sociale animale aux sociétés humaines. Le langage complexifie tout. Mais il reste que nous sommes des animaux, porteurs de tendances héritées de l'évolution, et qu'au niveau de généralité de la question posée par Michel Serres sur l'appropriation du savoir par le pouvoir, nous avons intérêt à analyser et mesurer la portée de la règle de biologie sociale qui fait que la dominance, par elle-même, semble sociogène et favoriser la paix sociale. Seule une étude expérimentale pourrait vraiment trancher sur ce point et distinguer ce qui est cause de ce qui est effet, mais en attendant je propose de généraliser et reconnaître que toute société fonctionne, pour ainsi dire, à l'inégalité au sens où l'on dit que l'organisme "se nourrit" d'entropie négative. Notre démocratie politique, très imparfaite, n'a encore que deux siècles, et même s'il est vrai que les sociétés humaines occidentales ont parcouru beaucoup de chemin dans

6. C. Murchison, "The experimental measurement of a social hierarchy in Gallus domesticus. IV, loss of body weight under conditions of mild starvation as a function of social dominance". Journal of General Psychology, 1935, 12, p. 296312. 
la réduction des inégalités les plus criantes, cette progression peut se maintenir et demeurer sans fin. Dans tous les cas ses acquis demeurent fragiles et réversibles. Si un jour la société humaine cesse d'être une société d'inégalité, la chose finira bien par se savoir, allez. Les avantages inhérents à la vie sociale sont tels que le plus mal loti des coopérants, tel Socrate après sa condamnation, préfère l'appartenance à l'exil.

4. Le pouvoir. L'inégalité engendre le Pouvoir, au double sens de la mécanique classique et de la politique humaine. En mécanique on appelle pouvoir ou potentiel l'énergie disponible pour produire du travail mesuré en ergs ou en joules. L'énergie est un réservoir de travail mécanique accumulé, comme un ressort sous tension, une charge électrique, un baril de poudre à canon, une chute d'eau. Cette énergie est apte à convertir cette inégalité en facteur de stabilité. C'est ce que fait voir une balance Roberval, dont le fléau est d'autant plus stable que ses deux plateaux sont chargés de poids plus inégaux. À l'opposé, à mesure qu'on égalise les deux poids, une charge additionnelle de plus en plus faible suffit pour miner la stabilité de l'ensemble, jusqu'à l'égalité totale qui maximise l'entropie du système. L'inégalité, c'est de l'ordre, au cœur de l'entropie négative et du pouvoir mécanique.

Je supposerai qu'il en va de même dans la vie sociale et politique, où la collaboration est possible quand la dominance des uns est assurée par l'altruisme, au sens biologique d'autosacrifice (comportement entraînant une baisse d'aptitude génétique individuelle), des autres. Je signale que cette proposition n'aura de valeur qu'intuitive tant qu'une recherche expérimentale sur les humains ne l'aura pas confirmée ou infirmée. Chez les non-humains la dominance est l'accès privilégié aux ressources comme la nourriture, l'espace y compris le nid, les occasions de reproduction. Sauf la "société parfaite», rarissime, qui sera décrite à l'instant, toutes les sociétés animales et humaines sont inégales à divers degrés. Aux fins de la construction de notre pont, le principal résultat de cette analyse est que, l'évolution nous ayant faits à la fois différents et sociaux, égoïstes et coopératifs, nous sommes incapables de vivre sans 
chefs. Les pires sont les sous-chefs, je sais. Mais n'allons pas cracher sur eux, bonnes gens, notre besoin d'avoir des chefs est à ce point absolu que par mauvais temps n'importe lequel d'entre nous peut faire l'affaire. La preuve. Apprenons plutôt la construction des ponts.

5. La société parfaite. Le nec plus ultra de la vie sociale semble atteint chez certains invertébrés de colonie comme le siphonophore, dont il existe plus de 300 espèces. Le Nanomia cara décrit par $\mathrm{E}$. $\mathrm{O}$. Wilson ${ }^{7}$ est un animal marin semblable à la méduse, composé d'un grand nombre d'individus extrêmement spécialisés qui se développent en colonie, les uns devenant des nectophores qui assurent les déplacements de la colonie en aspirant et rejetant l'eau dans une direction donnée, d'autres deviennent des organes reproducteurs, d'autres encore forment les tentacules qui saisissent la proie, d'autres la dévorent, d'autres pompent la nourriture le long de la longue queue jusqu'à son extrémité supérieure où un organisme individuel s'est gonflé de gaz et sert d'organe de flottaison à l'ensemble. Si l'on touche à la colonie à l'une de ses extrémités, elle fuit dans la direction opposée, à la manière d'un seul organisme. Par certains attributs le Nanomia cara ressemble à une colonie composée d'individus séparés, comme une colonie de fourmis, alors que son comportement global le fait plutôt considérer comme un seul organisme. Le biologiste finit par admettre qu'il est l'un et l'autre, organisme et colonie. On a là ce qui est probablement l'exemple le plus perfectionné d'intégration sociale égalitariste, dépourvu de hiérarchie sociale, où ce qu'on appelle le pouvoir n'existe pas et où la collaboration requise de chaque membre semble également bénéfique à tous. Une coopération sans inégalité. Tout révolutionnaire qui vaut son sel ne devrait-il pas travailler à la création d'une société de ce genre? La réponse est non, pour deux raisons. D'abord parce que chaque élément individuel de Nanomia cara, provenant du même œuf fertilisé, est génétiquement identique à tous les autres, c'est pourquoi il est évolutivement indifférent que l'un se reproduise

7. Edward O. Wilson, Sociobiology. The New Synthesis, Cambridge, Mass. : Harvard University Press, p. 384. 
et l'autre pas. Cette voie est interdite aux humains reproduits sexuellement. En deuxième lieu, nous ne voudrions pas de ce genre de vie même si elle nous était offerte parce qu'elle n'assure aucune aire de développement à l'individualisme prononcé rendu possible par la complexité de l'organisme humain. C'est une règle en biologie que l'autonomie va de pair avec la complexité : «Plus un organisme est complexe, plus il est libre8». Si ces détails avaient été connus en 1917 un savant aurait pu prédire à Lénine, qui n'en aurait probablement pas tenu compte, l'inévitable échec de sa révolution. II y a là une précieuse leçon de choses. Seule la biologie nous permet de comprendre que la société parfaite existe et que nous n'en voulons pas.

Le savoir, c'est de la vie. Nous ne connaissons la nature que par les échanges et transactions que nous faisons avec elle, qui la font et la transforment pendant qu'elle nous informe et nous fait. Le tournesol que j'ai planté oriente sa grosse tête vers le soleil et je penche la mienne sur ma page en écrivant ces lignes, tous deux avons besoin d'eau $\left(\mathrm{H}_{2} \mathrm{O}\right)$ et produisons du dioxyde de carbone $\left(\mathrm{CO}_{2}\right)$, j'alimente mon cerveau de l'oxygène $\left(\mathrm{O}_{2}\right)$ qu'il excrète et de ce qu'il m'apprend quand je l'interroge. Les protéines végétales qu'il sait produire font l'ordinaire des prédateurs herbivores dont je suis, incapables d'en produire pour leur compte, mon savoir à moi prend appui sur sa production et s'incorpore aux sociétés humaines qui le rendent possible. II n'y a rien, ici, qui n'irradie la liberté liée à la complexité de la vie dont parle François Jacob.

Je pense avoir répondu à la question «la plus rare et la plus évitée" posée par Michel Serres. Le savoir est asservi au pouvoir parce que nous sommes des animaux sociaux coopérant à l'intérieur des hiérarchies qui assurent notre paix sociale. II existe effectivement une libido dominandi, le mot est plus joli que la chose, obscurément mais effectivement liée à la libido tout court. Du pouvoir, on peut dire ce que l'on dit du sexe, tout n'y est pas réductible mais rien de ce que nous sommes ne lui

8. François Jacob, La logique du vivant. Une histoire de l'hérédité, Paris :

Gallimard, 1970, p. 207. 
échappe entièrement. Pour la société, le pouvoir est la lumière que nous ne pouvons pas voir mais grâce à laquelle nous apercevons ce que la société donne à voir d'elle-même. Pour l'individu, le pouvoir c'est les autres, la besace de devant :

\section{Le Fabricateur souverain}

Nous créa Besaciers tous de même manière,

Tant ceux du temps passé que du temps d'aujourd'hui.

II fit pour nos défauts la poche de derrière

Et celle de devant pour les défauts d'autrui.

\section{La Fontaine, La besace}

\section{La morale tribale}

II reste à comprendre la nature propre de cet asservissement du savoir au pouvoir afin de prendre la mesure de la difficulté qui attend celui qui voudrait l'abolir. J'ai montré ailleurs ${ }^{9}$ que la morale humaine est d'abord basée sur le groupe avant même l'entrée en jeu du libre arbitre individuel. Ce qui veut dire que l'interprétation du groupe oblige et délimite toujours celle de l'individu. Par exemple, il n'y a pas de libre arbitre chez Aristote, en l'absence du concept de sujet qui n'apparaîtra qu'en 1781 avec Kant (il n'y a pas davantage de sujet chez Descartes). Pourtant on ne trouve pas un seul commentaire de l'Éthique à Nicomaque d'Aristote qui lui donne tout son sens en l'illustrant par des exemples empruntés à la vie quotidienne de la démocratie athénienne de son temps, qu'en langage moderne on est bien obligé d'appeler un totalitarisme. Car on sait que le statut de Citoyen athénien était réservé à quelques centaines ou milliers de mâles, ce qui excluait d'un seul coup la moitié de la population athénienne, les femmes, plus quelque $80 \%$ des autres mâles, et les prêtres étaient des magistrats. Saurons-nous jamais ce qu'était la morale d'Aristote, de son propre point de vue de libre citoyen esclavagiste? Je pense qu'il nous est pratiquement impossible de rendre justice à un sys-

9. Les fondements biologiques de la morale. Montréal : Éditions les Herbes rouges, 1996. 
tème moral à moins qu'il soit fortement imprégné du rationalisme occidental, de la doctrine chrétienne du sujet individuel, du salut d'une âme immortelle et du libre arbitre dont Aristote n'avait pas la moindre idée. Ce n'est pas seulement la théorie morale qui nous est imposée par notre groupe avant tout jugement individuel, tout ce que nous prétendons savoir se trouve obéré de la sorte.

Les animaux vivant en société forment des groupes. Chez les humains, étant donné la dimension supplémentaire apportée par le langage, leurs groupes sont des tribus, au sens du biologiste Garrett Hardin'10. Tout groupe humain qui se considère lui-même et qui est considéré de l'extérieur comme étant distinct peut être appelé une tribu. Ce peut être une race ou une ethnie, mais ce peut aussi bien être une occupation, une profession ou une religion. La caractéristique essentielle de la tribu est son double standard de moralité : elle adopte un type de comportement à l'égard de ses propres membres, et un autre à l'égard de quiconque n'en est pas. Nous ne jugeons pas la morale aristotélicienne, qui est au principe même de la morale occidentale, selon les critères qui prévalaient en son temps, mais selon les nôtres, post-kantiens. Les hellénistes, principaux artisans en cette matière, n'y peuvent rien parce qu'eux-mêmes sont une tribu et qu'ils sont des nôtres.

Les savants, en général, sont aussi une tribu. Comment, dès lors, pourrait-on les persuader de dissocier leur savoir du pouvoir généralement fort limité qu'il leur assure individuellement? L'idée de tribalisme n'est ici qu'un élargissement, aux dimensions de la nature humaine, de ce que nous avons l'habitude d'appeler l'ethnocentrisme et qui n'est pas la xénophobie, seulement son antichambre ou la porte à côté. Toutes nos sciences humaines sont infléchies par cet ethnocentrisme et c'est pourquoi elles ne sont jamais devenues de véritables sciences comme elles l'avaient promis à leur naissance, quand elles prétendaient remplacer la philosophie. Qui ne se souvient de l'époque où la "critique des idéologies" prétendait supplanter la morale, rien de moins? II n'y a pas de sciences 10. Ibid., p. 124. 
humaines, seulement une philosophie améliorée qui tient enfin compte de la spécifité du social. L'ethnocentrisme des sciences humaines triomphe à ce point qu'à l'intérieur de sa seule version américaine, l'anthropologie, qui nous a donné le mot ethnocentrisme, compte maintenant une bonne vingtaine de spécialisations qui ne s'ignorent pas toujours, tant s'en faut, seulement tant qu'elles peuvent. Chacune semble avoir vocation tribale, indéfiniment ramifiable. Ce qui rendait le grand anthropologue anglais Victor Turner très malheureux.

La seule discipline qui échappe manifestement à l'ethnocentrisme est la mathématique qui, bien qu'elle ne soit pas ellemême une science, est au cœur de la science moderne. Une science est dite «exacte» ou "dure» dans la mesure où elle répond à sa mathématisation. Personne ne nous a expliqué pourquoi. Je soupçonne que l'universalité du langage mathématique est liée à l'unicité de l'architecture du cerveau humain. II n'y a qu'une espèce humaine. La clé de l'énigme serait alors à chercher en biologie.

Je prédis que nous demeurerons pratiquement sans défense contre les conséquences les plus fâcheuses de l'appropriation du savoir par le pouvoir, concentrées dans l'ethnocentrisme et la xénophobie, tant que nous refuserons de voir les fondements biologiques de la morale. Le pouvoir n'est pas que politique, il est aussi animal, je veux dire tribal. II est de bonne méthode de mettre à contribution la science expérimentale là où la philosophie est impuissante. À défaut de quoi l'ultime réplique aux pires méfaits de la morale tribale demeure la trahison'11.

Avec Hölderlin, il faut retourner le désir de quitter ce monde pour un autre en un désir de quitter l'autre pour celui-ci.

\section{Claude Lagadec Philosophe}

11. "Éloge de la trahison", dans Les fondements biologiques de la morale, pages 194-196. 